\title{
HOT, ROTATING DISKS IN GENERAL RELATIVITY: COLLISIONLESS EQUILIBRIUM MODELS
}

\author{
A. Katrin Schenk, ${ }^{1}$ Stuart L. Shapiro, ${ }^{2,3}$ AND Saul A. Teukolsky ${ }^{1,4}$ \\ Received 1998 January 15 ; accepted 1999 March 16
}

\begin{abstract}
We present a method for constructing equilibrium disks with net angular momentum in general relativity. The method solves the relativistic Vlasov equation coupled to Einstein's equations for the gravitational field. We apply the method to construct disks that are relativistic versions of Newtonian Kalnajs disks. In Newtonian gravity these disks are analytic and are stable against ring formation for certain ranges of their velocity dispersion. We investigate the existence of fully general relativistic equilibrium sequences for differing values of the velocity dispersion. These models are the first rotating, relativistic disk solutions of the collisionless Boltzmann equation.
\end{abstract}

Subject headings: accretion, accretion disks - relativity

\section{INTRODUCTION}

Rotating stellar disks in dynamical equilibrium have a long history in astrophysics. Such systems are described by self-consistent solutions to the Vlasov equation for the phase-space distribution function $f$ coupled to the equations for the gravitational field. Even in Newtonian gravitation, finding solutions is difficult since the configurations are nonspherical and have a relatively large number of nontrivial phase-space degrees of freedom. (For a general review and discussion, see Fridman \& Polyachenko 1984 or Binney \& Tremaine 1987.) When the configuration is relativistic, the gravitational field is described by Einstein's equations, and hence the problem is even more difficult.

In a previous paper (Shapiro \& Teukolsky 1993, hereafter Paper I), we developed a method for constructing equilibrium axisymmetric star clusters with net rotation in general relativity. In this paper we adapt this method to treat disks. Once again we restrict our attention to the simplest phase-space distribution functions that can give rise to nonspherical equilibria, functions of particle energy $E$, and angular momentum $J_{z}$ alone. Because $E$ and $J_{z}$ are integrals of the motion, choosing a distribution function of the form $f=f\left(E, J_{z}\right)$ guarantees that we will have a solution to the Vlasov equation, provided the metric is determined selfconsistently. No further dynamical equations need to be solved for the matter. By contrast, in equilibrium fluid systems one must integrate the equation of hydrostatic equilibrium.

Several researchers have studied the properties of selfgravitating fluid disks in general relativity. Morgan \& Morgan (1969) presented an analytic model of a fluid disk with no net angular momentum. This work was followed by Bardeen \& Wagoner (1969), who studied rapidly rotating, semianalytic, zero-pressure models, and by Salpeter \& Wagoner (1971), who looked at rapidly rotating fluid disks with some thickness. The model presented by Bardeen \& Wagoner (1969) was solved analytically in a series of papers

\footnotetext{
${ }^{1}$ Center for Radiophysics and Space Research, Cornell University, Ithaca, NY 14853.

${ }^{2}$ Department of Physics, University of Illinois at Urbana-Champaign, Urbana, IL 61801.

${ }^{3}$ Department of Astronomy and NCSA, University of Illinois at Urbana-Champaign, Urbana, IL 61801.

${ }^{4}$ Departments of Physics and Astronomy, Cornell University.
}

(Neugebauer \& Meinel 1993, 1994, 1995). This model was extended to include internal pressure and solved numerically by Kley (1997). Collisionless disks with no net angular momentum have been modeled and evolved using a particle simulation code by Abrahams, Shapiro, \& Teukolsky (1994). The work here presents the first fully general relativistic, rotating, collisionless disk models.

As an illustration of our method, we will focus on the relativistic generalization of an important class of Newtonian disks, the Kalnajs disks (Kalnajs 1972; Binney \& Tremaine 1987). These disks are completely described by simple analytic expressions. In the limiting case that all the particles move in circular orbits, the angular velocity and surface density are just those of the corresponding fluid Maclaurin spheroid in the disk limit (eccentricity $\rightarrow 1$ ). In Newtonian theory, however, it is known that equilibrium disks supported against collapse by rotation alone are unstable to ring formation (see, e.g., § 5.3 of Binney \& Tremaine 1987). Kalnajs (1972) showed that the disk can be stabilized by "heating" it, that is, converting some of the ordered rotational energy into random thermal motion while keeping the surface density the same. (Properties of Newtonian Kalnajs disks are reviewed in the Appendix.) We construct relativistic generalizations of Kalnajs disks and study their properties.

In addition to its potential astrophysical significance for generating models of highly relativistic stellar disks or collisionless particle distributions, the method presented here provides a class of rotating equilibria that has not been treated previously in the general relativistic literature. These collisionless models join Kerr black holes and rotating fluid stars and fluid disks as physically realistic rotating equilibria in general relativity. Such solutions to Einstein's equations provide important insight into the effects of rotation in a strong gravitational field. They also provide useful initial data for evolution codes in general relativity. In particular, such codes can assess the stability of relativistic Kalnajs disks, since, as we will see below, we can only make heuristic statements about their stability from the results of this paper.

\section{BASIC EQUATIONS}

In this paper we consider rotating equilibrium stellar disks that are axisymmetric. The metric can then be written 
in the form

$$
\begin{aligned}
d s^{2}= & -e^{\gamma+\rho} d t^{2}+e^{2 \sigma}\left(d r^{2}+r^{2} d \theta^{2}\right) \\
& +e^{\gamma-\rho} r^{2} \sin ^{2} \theta(d \phi-\omega d t)^{2},
\end{aligned}
$$

where the metric potentials $\rho, \gamma, \omega$, and $\sigma$ are functions of $r$ and $\theta$ only. Here and throughout we set $G=c=1$. This is the same form of the metric used in Paper I.

All calculations in this paper are performed in the Zero Angular Momentum Observer (ZAMO) frame (Bardeen 1970; Lightman et al. 1975). This is the orthonormal frame of a zero angular momentum observer, whose basis 1 forms $\omega^{\alpha}$ are related to the coordinate basis $d x^{\alpha}$ by

$$
\omega^{\hat{\alpha}}=L_{\alpha}^{\hat{\alpha}} d x^{\alpha},
$$

where

$$
L_{\alpha}^{\hat{\alpha}}=\left[\begin{array}{cccc}
e^{v} & 0 & 0 & 0 \\
0 & e^{v} & 0 & 0 \\
0 & 0 & r e^{v} & 0 \\
-\omega r \sin \theta e^{\beta} & 0 & 0 & r \sin \theta e^{\beta}
\end{array}\right],
$$

and where

$$
\begin{aligned}
& v=\frac{\gamma+\rho}{2}, \\
& \beta=\frac{\gamma-\rho}{2} .
\end{aligned}
$$

The corresponding orthonormal basis vectors are given by

$$
\boldsymbol{e}_{\hat{\alpha}}=L_{\hat{\alpha}}^{\alpha} \frac{\partial}{\partial x^{\alpha}},
$$

where $L_{\hat{\alpha}}^{\alpha}$ is the inverse matrix of $L_{\alpha}^{\hat{\alpha}}$.

Following Komatsu, Eriguchi, \& Hachisu (1989), Cook, Shapiro, \& Teukolsky (1992), and Paper I, we write the Einstein field equations that determine $\rho, \gamma$, and $\omega$ in the form

$$
\begin{gathered}
\nabla^{2}\left(\rho e^{\gamma / 2}\right)=S_{\rho}(r, \mu), \\
\left(\nabla^{2}+\frac{1}{r} \partial_{r}-\frac{\mu}{r^{2}} \partial_{\mu}\right)\left(\gamma e^{\gamma / 2}\right)=S_{\gamma}(r, \mu), \\
\left(\nabla^{2}+\frac{2}{r} \partial_{r}-\frac{2 \mu}{r^{2}} \partial_{\mu}\right)\left(\omega e^{(\gamma-2 \rho) / 2}\right)=S_{\omega}(r, \mu),
\end{gathered}
$$

where $\nabla^{2}$ is the flat-space, spherical coordinate scalar Laplacian, $\mu=\cos \theta$, and $S_{\rho}, S_{\gamma}$, and $S_{\omega}$ are effective source terms that include the nonlinear and matter terms. In the equations below, we explicitly exhibit the disk nature of the matter by defining a disk stress-stress-energy tensor, $t_{\hat{\nu}}^{\hat{\mu}}(r)$, such that the full stress-energy tensor, $T_{\hat{v}}^{\hat{\mu}}(r, \mu)$, is given by

$$
T_{\hat{\nu}}^{\hat{\mu}}(r, \mu)=t_{\hat{v}}^{\hat{\mu}}(r) \frac{\delta(\mu)}{r} .
$$

Thus the integrals used to calculate the stress-energy components, equations (31)-(33) below, need only be evaluated in the disk plane $(\mu=0)$.

The effective source terms are given by

$$
S_{\rho}(r, \mu) \equiv R_{\rho}(r, \mu)+t_{\rho}(r) \frac{\delta(\mu)}{r},
$$

$$
\begin{gathered}
S_{\gamma}(r, \mu) \equiv R_{\gamma}(r, \mu)+t_{\gamma}(r) \frac{\delta(\mu)}{r}, \\
S_{\omega}(r, \mu) \equiv R_{\omega}(r, \mu)+t_{\omega}(r) \frac{\delta(\mu)}{r},
\end{gathered}
$$

where we have defined matter-dependent and matterindependent source terms such that

$$
\begin{aligned}
& \boldsymbol{R}_{\rho}(r, \mu)=e^{\gamma / 2}\left\{\frac{1}{r} \gamma_{, r}-\frac{\mu}{r^{2}} \gamma_{, \mu}+\frac{\rho}{2}\left[-\gamma_{, r}\left(\frac{1}{2} \gamma_{, r}+\frac{1}{r}\right)\right.\right. \\
& \left.-\frac{1}{r^{2}} \gamma_{, \mu}\left(\frac{1-\mu^{2}}{2} \gamma_{, \mu}-\mu\right)\right] \\
& \left.+r^{2}\left(1-\mu^{2}\right) e^{-2 \rho}\left(\omega_{, r}^{2}+\frac{1-\mu^{2}}{r^{2}} \omega_{, \mu}^{2}\right)\right\}, \\
& t_{\rho}(r, \mu)=8 \pi e^{\gamma / 2} e^{2 \sigma}\left[t_{\hat{\phi}}^{\hat{\phi}}-t_{\hat{t}}^{\hat{r}}+\frac{\rho}{2}\left(t_{\hat{r}}^{\hat{r}}+t_{\hat{\theta}}^{\hat{\theta}}\right)\right], \\
& R_{\gamma}(r, \mu)=e^{\gamma / 2} \frac{\gamma}{2}\left(-\frac{1}{2} \gamma_{, r}^{2}-\frac{1-\mu^{2}}{2 r^{2}} \gamma_{, \mu}^{2}\right), \\
& t_{\gamma}(r, \mu)=8 \pi e^{\gamma / 2} e^{2 \sigma}\left(1+\frac{\gamma}{2}\right)\left(t_{\hat{r}}^{\hat{r}}+t_{\hat{\theta}}^{\hat{\hat{\vartheta}}}\right), \\
& R_{\omega}(r, \mu)=e^{(\gamma-2 \rho) / 2} \omega\left[-\frac{1}{r}\left(2 \rho_{, r}+\frac{1}{2} \gamma_{, r}\right)\right. \\
& \left.+\frac{\mu}{r^{2}}\left(2 \rho_{, \mu}+\frac{1}{2} \gamma_{, \mu}\right)+\frac{1}{4}\left(4 \rho_{, r}^{2}-\gamma_{, r}^{2}\right)\right] \\
& +\frac{1-\mu^{2}}{4 r^{2}}\left(4 \rho_{, \mu}^{2}-\gamma_{, \mu}^{2}\right)-r^{2}\left(1-\mu^{2}\right) e^{-2 \rho} \\
& \left.\times\left(\omega_{, r}^{2}+\frac{1-\mu^{2}}{r^{2}} \omega_{, \mu}^{2}\right)\right], \\
& t_{\omega}(r, \mu)=8 \pi e^{(\gamma-2 \rho) / 2} e^{2 \sigma} \\
& \times\left[-\omega\left(t_{\hat{\phi}}^{\hat{\phi}}-t_{\hat{t}}^{\hat{\hat{t}}}\right)+\frac{\omega}{2}\left(t_{\hat{r}}^{\hat{r}}+t_{\hat{\theta}}^{\hat{\theta}}\right)-\frac{2 e^{\rho} t_{\hat{\phi}}^{\hat{\phi}}}{r\left(1-\mu^{2}\right)^{1 / 2}}\right] .
\end{aligned}
$$

Here $t_{\hat{v}}^{\hat{\mu}}$ are the orthonormal components of our disk stressenergy tensor for collisionless matter in the ZAMO frame (see below).

The fourth field equation determines $\sigma$ and is given by

$$
\begin{aligned}
\sigma_{, \mu}= & -\frac{1}{2}\left(\rho_{, \mu}+\gamma_{, \mu}\right) \\
& -\left\{\left(1-\mu^{2}\right)\left(1+r \gamma_{, r}\right)^{2}+\left[\mu-\left(1-\mu^{2}\right) \gamma_{, \mu}\right]^{2}\right\}^{-1} \\
& \times\left(\frac{1}{2}\left[r^{2}\left(\gamma_{, r r}+\gamma_{, r}^{2}\right)-\left(1-\mu^{2}\right)\left(\gamma_{, \mu \mu}+\gamma_{, \mu}^{2}\right)\right]\right. \\
& \times\left[-\mu+\left(1-\mu^{2}\right) \gamma_{, \mu}\right] \\
& +r \gamma_{, r}\left[\frac{1}{2} \mu+\mu r \gamma_{, r}+\frac{1}{2}\left(1-\mu^{2}\right) \gamma_{, \mu}\right] \\
& +\frac{3}{2} \gamma_{, \mu}\left[-\mu^{2}+\mu\left(1-\mu^{2}\right) \gamma_{, \mu}\right] \\
& -r\left(1-\mu^{2}\right)\left(\gamma_{, r \mu}+\gamma_{, r} \gamma_{, \mu}\right)\left(1+r \gamma_{, r}\right) \\
& -\frac{1}{4} \mu r^{2}\left(\rho_{, r}+\gamma_{, r}\right)^{2}
\end{aligned}
$$




$$
\begin{aligned}
& -\frac{r}{2}\left(1-\mu^{2}\right)\left(\rho_{, r}+\gamma_{, r}\right)\left(\rho_{, \mu}+\gamma_{, \mu}\right) \\
& +\frac{1}{4} \mu\left(1-\mu^{2}\right)\left(\rho_{, \mu}+\gamma_{, \mu}\right)^{2} \\
& -\frac{r^{2}}{2}\left(1-\mu^{2}\right) \gamma_{, r}\left(\rho_{, r}+\gamma_{, r}\right)\left(\rho_{, \mu}+\gamma_{, \mu}\right) \\
& +\frac{1}{4}\left(1-\mu^{2}\right) \gamma_{, \mu}\left[r^{2}\left(\rho_{, r}+\gamma_{, r}\right)^{2}-\left(1-\mu^{2}\right)\left(\rho_{, \mu}+\gamma_{, \mu}\right)^{2}\right] \\
& +\left(1-\mu^{2}\right) e^{-2 \rho}\left\{\frac{1}{4} r^{4} \mu \omega_{, r}^{2}+\frac{1}{2} r^{3}\left(1-\mu^{2}\right) \omega_{, r} \omega_{, \mu}\right. \\
& -\frac{1}{4} r^{2} \mu\left(1-\mu^{2}\right) \omega_{, \mu}^{2} \\
& +\frac{1}{2} r^{4}\left(1-\mu^{2}\right) \gamma_{, r} \omega_{, r} \omega_{, \mu} \\
& \left.-\frac{1}{4} r^{2}\left(1-\mu^{2}\right) \gamma_{, \mu}\left[r^{2} \omega_{, r}^{2}-\left(1-\mu^{2}\right) \omega_{, \mu}^{2}\right]\right\} \\
& -r^{2}\left[\mu-\left(1-\mu^{2}\right) \gamma_{, \mu}\right] e^{2 \sigma} 4 \pi\left(t_{\hat{r}}^{\hat{r}}-t_{\hat{\theta}}^{\hat{\vartheta}}\right) \frac{\delta(\mu)}{r} \\
& \left.+r^{2}\left(1-\mu^{2}\right)^{1 / 2}\left(1+r \gamma_{, r}\right) e^{2 \sigma} 8 \pi t_{\hat{\theta}}^{\hat{\ell}} \frac{\delta(\mu)}{r}\right) .
\end{aligned}
$$

The terms containing $t_{\hat{\theta}}^{\hat{\theta}}$ and $t_{\hat{\theta}}^{\hat{\theta}}$ in the above equation are identically zero for an equatorial disk since each particle has no $\theta$-component of momentum. The remaining matter term in equation (20),

$$
-r^{2}\left[\mu-\left(1-\mu^{2}\right) \gamma, \mu\right] e^{2 \sigma} 4 \pi t_{\hat{p}}^{\hat{r}} \frac{\delta(\mu)}{r},
$$

also does not contribute since $\left.\gamma_{\mu}\right|_{\mu=0}$ vanishes by symmetry.

The stress-energy tensor for the matter is determined by the phase-space distribution function $f$, which is governed by the relativistic Vlasov equation (the matter-dynamical equation). Any distribution function of the form $f=f\left(E, J_{z}\right)$ is an equilibrium solution of the Vlasov equation in axisymmetry. Here $E$ and $J_{z}$ are the two constants of motion associated with the Killing vectors $\partial / \partial t$ and $\partial / \partial \phi$. For our two-dimensional disk system, they are

$$
\begin{gathered}
E \equiv-p \cdot \frac{\partial}{\partial t}=e^{v_{e}} p^{\hat{t}}+\omega e^{\beta_{e}} r p^{\hat{\phi}}, \\
J_{z} \equiv p \cdot \frac{\partial}{\partial \phi}=e^{\beta_{e}} r p^{\hat{\phi}},
\end{gathered}
$$

where $p^{\hat{\alpha}}$ are the orthonormal components of the particle 4-momentum $p$ in the ZAMO frame, and we have defined, for convenience, the quantities

$$
\begin{aligned}
v_{e} & \equiv v(r, 0), \\
\beta_{e} & \equiv \beta(r, 0), \\
\omega_{e} & \equiv \omega(r, 0), \quad \text { etc } .
\end{aligned}
$$

Physically, $E$ is the conserved energy of a particle, and $J_{z}$ is the conserved angular momentum about the symmetry axis.
Working in two dimensions, we define the disk stressenergy tensor for the matter,

$$
t^{\hat{\alpha} \hat{\beta}}=\int f p^{\hat{\alpha}} p^{\hat{\beta}} \frac{d^{2} \hat{p}}{p^{\hat{t}}} .
$$

Here, for a disk, we have

$$
\begin{gathered}
p^{\hat{t}}=\left[\left(p^{\hat{\gamma}}\right)^{2}+\left(p^{\hat{\phi}}\right)^{2}+m^{2}\right]^{1 / 2}, \\
d^{2} \hat{p}=d p^{\hat{p}} d p^{\hat{\phi}},
\end{gathered}
$$

where $m$ is the particle mass. For the field equations (7)-(9) and (20), we need only the combinations

$$
\begin{gathered}
t_{1} \equiv t_{\phi}^{\hat{\phi}}-t_{\hat{t}}^{\hat{t}}, \\
t_{2} \equiv t_{\hat{\mathrm{r}}}^{\hat{\gamma}}, \\
t_{03} \equiv t_{\hat{\phi}}^{\hat{t}} .
\end{gathered}
$$

Using equations (25)-(27), we get

$$
\begin{gathered}
t_{1}(r)=\int d p^{\hat{\gamma}} \int d p^{\hat{\phi}} \frac{\left(p^{\hat{p}}\right)^{2}+2\left(p^{\hat{\phi}}\right)^{2}+m^{2}}{\left[\left(p^{\hat{\gamma}}\right)^{2}+\left(p^{\hat{\phi}}\right)^{2}+m^{2}\right]^{1 / 2}} f\left(E, J_{z}\right), \\
t_{2}(r)=\int d p^{\hat{\hat{p}}} \int d p^{\hat{\phi}} \frac{\left(p^{\hat{r}}\right)^{2}}{\left[\left(p^{\hat{\gamma}}\right)^{2}+\left(p^{\hat{\phi}}\right)^{2}+m^{2}\right]^{1 / 2}} f\left(E, J_{z}\right), \\
t_{03}(r)=\int d p^{\hat{r}} \int d p^{\hat{\phi}} p^{\hat{\phi}} f\left(E, J_{z}\right) .
\end{gathered}
$$

At each spatial point $(r, \mu)$, the limits of integration in equations (31)-(33) will be determined by the distribution function, as discussed in the next subsection.

\subsection{Distribution Function}

The Newtonian Kalnajs distribution function is given by equation (A4). We construct a relativistic generalization by letting $E_{\mathrm{N}} \rightarrow E-m$ :

$$
\begin{aligned}
& f_{\mathrm{GR}}\left(E, J_{z}\right)= \\
& \begin{cases}K\left\{2\left[\left(b V J_{z} / R_{m}\right)-(E-m)\right]-V^{2}\left(1+b^{2}\right)\right\}^{-1 / 2}, & {[\cdots]>0,} \\
0, & {[\cdots] \leq 0 .}\end{cases}
\end{aligned}
$$

Here $V, b$, and $R_{m}$ are constants, which in the Newtonian limit have the following interpretations:

$$
b \equiv \frac{\text { Mean angular rotation rate }}{\text { Angular speed of a circular orbit }} \equiv \frac{\Omega}{\Omega_{\text {circ }}} \equiv \frac{\Omega R_{m}}{V},
$$

and $R_{m}$ is the matter radius. Note that in the Newtonian limit we have

$$
E-m \rightarrow E_{\mathrm{N}}, \quad J_{z} \rightarrow J_{z \mathrm{~N}},
$$

where $E_{\mathrm{N}}$ and $J_{z \mathrm{~N}}$ are the Newtonian energy and angular momentum as defined by equations (A5) and (A6). Thus we recover the Newtonian distribution function $f_{\mathrm{N}}$, equation (A4), from equation (34) in the Newtonian limit.

Since the distribution function is nonzero only for positive values of the argument of the square root, we can solve for the limits of integration for the stress-energy integrals (eqs. [31]-[33]) by using the expressions (22) and (23) for $E$ and $J_{z}$ in equation (34) and setting the argument of the 
square root to zero. Thus we find the limits to be

$$
\begin{aligned}
& 0<p^{\hat{r}}<p_{\max }^{\hat{r}}\left(p^{\hat{\phi}}, r\right) \\
& p_{-}^{\hat{\phi}}(r)<p^{\hat{\phi}}<p_{+}^{\hat{\phi}_{+}}(r),
\end{aligned}
$$

with

$$
\begin{gathered}
p_{\max }^{\hat{r}}\left(p^{\hat{\phi}}, r\right) \equiv\left[\frac{a^{2}+d^{2}-1}{1-d^{2}}-\left(1-d^{2}\right)\left(p^{\hat{\phi}}-\frac{a d}{1-d^{2}}\right)^{2}\right]^{1 / 2} \\
p_{ \pm}^{\hat{\phi}} \equiv \frac{a d}{1-d^{2}} \pm \frac{\left(a^{2}+d^{2}-1\right)^{1 / 2}}{1-d^{2}}
\end{gathered}
$$

Here $a$ and $d$ are functions of $r$ only and are given by

$$
\begin{aligned}
& a(r) \equiv e^{-v_{e}(r)}\left[1-\frac{V^{2}}{2}\left(1-b^{2}\right)\right], \\
& d(r) \equiv e^{\beta_{e}(r)-v_{e}(r)} r\left[b \frac{V}{R_{m}}-\omega_{e}(r)\right] .
\end{aligned}
$$

Note that in Paper I the distribution functions considered contained a parameter $E_{\max }$ that was less than $m$ for bound systems of finite extent. The limits of integration were determined by $E \leq E_{\max }$. Here one can verify that the limits (37) and (38) never violate the condition $E \leq m$. Curiously, equality can be attained even in the Newtonian limit: $E_{\mathrm{N}} \rightarrow 0$ at the outer edge of a "cold" Kalnajs disk.

The total mass-energy of the system is

$$
\begin{aligned}
M & =-\int\left(2 T_{v}^{\mu}-\delta_{v}^{\mu} T\right) \xi_{(t)}^{v} d^{3} \Sigma_{\mu} \\
& =\int\left(-2 T_{t}^{t}+T\right) \sqrt{-g} d^{3} x .
\end{aligned}
$$

Here $\xi_{(t)}^{v}=\partial / \partial t$ is the time Killing vector. Transforming the integrand to the ZAMO frame, and integrating out the $\delta$-function in $\mu$, we obtain

$$
M=2 \pi \int\left[t_{1}(r)+t_{2}(r)+2 \omega_{e} e^{-\rho_{e}} r t_{03}(r)\right] e^{2 \sigma_{e}+\gamma_{e}} r d r
$$

As we will see in $\S 5$, this equation plays a crucial role in the iterative solution of the combined matter and field equations.

\section{DIAGNOSTIC PROBES}

There are a number of useful quantities that characterize an equilibrium system once a solution has been obtained. The total angular momentum is given by

$$
\begin{aligned}
J & =\int T_{v}^{\mu} \xi_{(\phi)}^{v} d^{3} \Sigma_{\mu} \\
& =\int T_{\phi}^{t} \sqrt{-g} d^{3} x .
\end{aligned}
$$

Transforming to the ZAMO frame, and again integrating out the $\delta$-function in $\mu$, we get

$$
J=2 \pi \int t_{03}(r) e^{2\left(\sigma_{e}+\beta_{e}\right)} r^{2} d r .
$$

The surface rest mass density is given by

$$
\sigma_{0}(r)=m \int d p^{\hat{r}} \int d p^{\hat{\phi}} f_{\mathrm{GR}}\left(E, J_{z}\right)
$$

and the total rest mass by

$$
M_{0}=2 \pi \int \sigma_{0}(r) e^{2 \sigma_{e}+\beta_{e}} r d r
$$

The binding energy of the system is defined as

$$
E_{b} \equiv M_{0}-M
$$

and can be computed from equations (44) and (48).

\section{SCALING AND NONDIMENSIONAL UNITS}

The quantities $m$ and $M$ can be scaled out of all the above equations. For example, we can define

$$
\begin{gathered}
\tilde{p}=p / m, \quad \tilde{r}=r / M, \quad \tilde{J}=J / M^{2}, \\
\tilde{t}^{\alpha \beta}=t^{\alpha \beta} / M^{-1}, \quad \tilde{f}_{\mathrm{GR}}=f_{\mathrm{GR}} / M^{-1} m^{-3}, \quad \tilde{J}_{z}=J_{z} / m M .
\end{gathered}
$$

With these definitions, all of the previous equations can be written in tilde variables without $m$ or $M$ appearing. Equivalently, the original equations can be solved setting $m=M=1$ and scaling the final results according to equation (50) to accommodate arbitrary values of $m$ and $M$. Henceforth we will make this simplification.

In the Newtonian limit there is an additional scale freedom in that we can also set $R_{m} / M$ to 1 . This is not the case for relativistic systems.

\section{NUMERICAL SOLUTION}

The numerical scheme used here is a two-dimensional analog of the procedure adopted in Paper I. In this procedure we start with initial guesses for the metric potentials, $\rho, \gamma, \omega$, and $\sigma$ (see $\S 6$ below). We then integrate equations (31)-(33) to find $t_{1}, t_{2}$, and $t_{03}$ up to the constant factor $K$ appearing in equation (34). This unknown factor $K$ is fixed by requiring that the total mass of the system, equation (44), satisfy $M=1$. Next we integrate the field equations (7) and (8) for $\rho, \gamma$, and $\omega$, and then equation (20) for $\sigma$. We then iterate this procedure until some convergence criterion is met.

Using a combination of integral and finite-differencing techniques, we solve the equations for the matter and gravitational fields. These equations are solved on a discrete grid in $\mu$ and $r$ on the computational domain $0 \leq r \leq \infty$ and $0 \leq \mu \leq 1$. Unlike the Newtonian case, we cannot restrict the computational domain to the matter interior because the matter-independent effective source terms $\boldsymbol{R}_{\rho}, \boldsymbol{R}_{\gamma}$, and $R_{\omega}$ are nonzero in the vacuum exterior. Consequently, we divide the radial grid into an interior and exterior domain. Each domain is covered by a geometrically spaced grid in $r$, with the grids joined smoothly at the cluster surface. We use an angular grid that is uniformly spaced in $\mu$. The interior radial grid is arranged to yield sufficient resolution for the core of the cluster, while the outer grid extends to some sufficiently large radius, typically 2-3 times the radius of the matter surface. High resolution of the core is crucial for obtaining numerical accuracy in highly centrally condensed relativistic disks.

The three elliptic field equations (7)-(9) are solved by an integral Green's function approach following Komatsu et 
al. (1989) and Cook et al. (1992). Again we make a matterdependent, matter-independent split of the Green's functions depending on whether we are integrating the matter-dependent source terms $t_{\rho}, t_{\gamma}$, and $t_{\omega}$, or the matterindependent source terms $\boldsymbol{R}_{\rho}, \boldsymbol{R}_{\gamma}$, and $\boldsymbol{R}_{\omega}$. Thus we write the solution of equation (7) as

$$
\rho(r, \mu)=W_{\rho}(r, \mu)+G_{\rho}(r, \mu),
$$

where we have defined

$$
\begin{aligned}
W_{\rho}(r, \mu) \equiv & -\sum_{n=0}^{\infty} e^{-\gamma / 2} \int_{0}^{\infty} d r^{\prime} \\
& \times \int_{0}^{1} d \mu^{\prime} r^{\prime 2} f_{2 n}^{2}\left(r, r^{\prime}\right) P_{2 n}(\mu) P_{2 n}\left(\mu^{\prime}\right) R_{\rho}\left(r^{\prime}, \mu^{\prime}\right), \\
G_{\rho}(r, \mu) \equiv & -\frac{1}{2} \sum_{n=0}^{\infty} e^{-\gamma / 2} P_{2 n}(\mu) \frac{(-1)^{n}(2 n-1) ! !}{2^{n} n !} \\
& \times \int_{0}^{\infty} d r^{\prime} r^{\prime} f_{2 n}^{2}\left(r, r^{\prime}\right) t_{\rho}\left(r^{\prime}, 0\right) .
\end{aligned}
$$

Here we have used the $\delta$-function in $\mu$ to carry out the $\mu^{\prime}$ integration in $G_{\rho}$. Similarly, to solve equation (8) we write

$$
r \sin \theta \gamma(r, \mu)=W_{\gamma}(r, \mu)+G_{\gamma}(r, \mu),
$$

with

$$
\begin{gathered}
W_{\gamma}(r, \mu) \equiv-\frac{2}{\pi} \sum_{n=1}^{\infty} e^{-\gamma / 2} \int_{0}^{\infty} d r^{\prime} \int_{0}^{1} d \mu^{\prime} r^{\prime 2} f_{2 n-1}^{1}\left(r, r^{\prime}\right) \\
\quad \times \frac{1}{2 n-1} \sin (2 n-1) \theta \sin (2 n-1) \theta^{\prime} R_{\gamma}\left(r^{\prime}, \mu^{\prime}\right) \\
G_{\gamma}(r, \mu) \equiv \frac{1}{\pi} \sum_{n=1}^{\infty} e^{-\gamma / 2} \frac{\sin (2 n-1) \theta(-1)^{n}}{(2 n-1)} \\
\times \int_{0}^{\infty} d r^{\prime} f_{2 n-1}^{1}\left(r, r^{\prime}\right) t_{\gamma}\left(r^{\prime}, 0\right)
\end{gathered}
$$

Finally, to solve equation (9) we write

$$
r \sin \theta \omega(r, \mu)=W_{\omega}(r, \mu)+G_{\omega}(r, \mu),
$$

with

$$
\begin{aligned}
W_{\omega}(r, \mu) \equiv & -\sum_{n=1}^{\infty} e^{(2 \rho-\gamma) / 2} \int_{0}^{\infty} d r^{\prime} \int_{0}^{1} d \mu^{\prime} r^{\prime 3} \sin \theta^{\prime} f_{2 n-1}^{2}\left(r, r^{\prime}\right) \\
& \times \frac{1}{2 n(2 n-1)} P_{2 n-1}^{1}(\mu) P_{2 n-1}^{1}\left(\mu^{\prime}\right) R_{\omega}\left(r^{\prime}, \mu^{\prime}\right) \\
G_{\omega}(r, \mu) \equiv & -\frac{1}{2} \sum_{n=1}^{\infty} e^{(2 \rho-\gamma) / 2} \frac{P_{2 n-1}^{1}(\mu)}{2 n(2 n-1)} \frac{(-1)^{n}(2 n-1) ! !}{2^{n}(n-1) !} \\
& \times \int_{0}^{\infty} d r^{\prime} r^{\prime 2} f_{2 n-1}^{2}\left(r, r^{\prime}\right) t_{\omega}\left(r^{\prime}, 0\right)
\end{aligned}
$$

Here

$$
\begin{gathered}
f_{n}^{1}\left(r, r^{\prime}\right)= \begin{cases}\left(r^{\prime} / r\right)^{n}, & \text { for } r^{\prime} / r \leq 1, \\
\left(r / r^{\prime}\right)^{n}, & \text { for } r^{\prime} / r>1,\end{cases} \\
f_{n}^{2}\left(r, r^{\prime}\right)= \begin{cases}(1 / r)\left(r^{\prime} / r\right)^{n}, & \text { for } r^{\prime} / r \leq 1, \\
\left(1 / r^{\prime}\right)\left(r / r^{\prime}\right)^{n}, & \text { for } r^{\prime} / r>1 .\end{cases}
\end{gathered}
$$

Among the advantages of this Green's function approach for solving the elliptic field equations is that the asymptotic conditions on $\rho, \gamma$, and $\omega$ are imposed automatically. That is, $\rho \sim O(1 / r), \gamma \sim O\left(1 / r^{2}\right)$, and $\omega \sim O\left(1 / r^{3}\right)$ for large $r$. To improve the accuracy of the angular integrations, we use the identities in equations (34)-(38) of Cook et al. (1992).

It should be noted that in the integration of equation (59), the calculation of $t_{\omega}(r, 0)$ at $r=0$ requires special care. It can be seen from equations (33), (39), and (40) that $t_{03}(r=$ $0)=0$, while regularity conditions near the axis imply that $t_{03} \sim r$ near $r=0$. Thus at $r=0$, the calculation of the quantity $t_{03} / r$ that appears in equation (19) is done by computing the quantity $d t_{03} /\left.d r\right|_{r=0}$ analytically. We do this by taking a derivative of equation (33) directly. For any $r$ this gives

$$
\begin{aligned}
& \frac{d t_{03}}{d r}=\frac{d}{d r}\left[\int_{p_{-}^{\hat{\phi}}(r)}^{p_{+}^{\hat{\phi}_{+}}(r)} d p^{\hat{\phi}} p^{\hat{\phi}}\right. \\
& \left.\times \int_{0}^{p_{\max }^{\hat{\mathrm{f}}}\left(r, p^{\hat{\phi})}\right.} d p^{\hat{\gamma}} f_{\mathrm{GR}}\left(E, J_{z}\right)\right] \\
& =\int_{p^{\underline{\hat{\phi}}}(r)}^{p_{\hat{\phi}}^{\hat{\phi}}(r)} d p^{\hat{\phi}} p^{\hat{\phi}} \frac{\partial I\left(p^{\hat{\phi}}, r\right)}{\partial r} \\
& +p_{+}^{\hat{\phi}_{+}}(r) I\left[p_{+}^{\hat{\phi}}(r), r\right] \frac{d p_{+}^{\hat{\phi}}(r)}{d r} \\
& -p_{-}^{\hat{\phi}}(r) I\left[p_{-}^{\hat{\phi}}(r), r\right] \frac{d p_{-}^{\hat{\phi}_{-}}(r)}{d r} .
\end{aligned}
$$

Here $p_{ \pm}^{\hat{\phi}}(r)$ and $p_{\max }^{\hat{r}}\left(r, p^{\hat{\phi}}\right)$ are defined in equations (40) and (39), and we have defined $I\left(p^{\hat{\phi}}, r\right)$ to be

$$
I\left(p^{\hat{\phi}}, r\right)=\int_{0}^{\hat{p}_{\max }^{\hat{p}}(r, p \hat{\phi})} d p^{\hat{p}} f_{\mathrm{GR}}\left(E, J_{z}\right) .
$$

At $r=0$ it can be shown that the first term in equation (63) vanishes by symmetry. The other two terms involve the integral $I\left(p^{\phi}, r\right)$ evaluated at $p^{\hat{\phi}}=p_{ \pm}^{\hat{\phi}}(0)$ and $r=0$. From equation (64) and the form of the distribution function $f_{\text {GR }}$ (eq. [34]), we see that this integrand is divergent and the limits collapse to zero at $p^{\hat{\phi}}=p_{ \pm}^{\hat{\phi}}(0)$. However, near $p^{\hat{\phi}}=$ $p_{ \pm}^{\hat{\phi}}(0)$ we can expand the integrand (and upper limit) to get an estimate of the value of the integral for use in the calculation of $d t_{03} /\left.d r\right|_{r=0}$. This yields

$$
\left.\frac{d t_{03}}{d r}\right|_{r=0}=\pi\left[\sqrt{a(r)\left[a^{2}(r)-1\right]} a(r) d_{, r}(r) e^{-v_{e} / 2}\right]_{r=0} .
$$

Here $a(r)$ and $d(r)$ are functions defined by equations (41) and (42).

Equation (20) for $\sigma$ is solved by integrating the linear ordinary differential equation from the pole $(\mu=1)$ to the equator with the initial condition that

$$
\sigma=\frac{\gamma-\rho}{2} \quad \text { at } \quad \mu=1,
$$

which arises from the requirement of local flatness on the coordinate axis. The derivatives of $\rho, \gamma$, and $\omega$ appearing in the matter-independent source terms $R_{\rho}, R_{\gamma}, R_{\omega}$, and the right-hand side of equation (20) are evaluated by finite differencing. 
Since we can evaluate the integrands in equations (31)(33) at any values of $p^{\hat{\gamma}}$ and $p^{\hat{\phi}}$, we carry out the quadrature over each variable by Romberg integration (Press et al. 1992).

In general, for convergent solutions we require the maximum fractional change in all four metric functions to be less than $1 \times 10^{-4}$ on successive iterations. However, much computing time can be saved by requiring runs to converge to only $1 \times 10^{-3}$. We have found that the change in physical quantities between solutions converged at the $1 \times 10^{-3}$ and $1 \times 10^{-4}$ levels is, at its largest, on the order of $0.8 \%$. We have also run selected cases with an even stricter convergence criterion and verified that the changes in the solution parameters are then even smaller than $0.8 \%$. Convergence to $1 \times 10^{-4}$ typically takes about $25-30$ iterations when we start with a Newtonian initial guess (§6). The ability of our code to converge was significantly enhanced by the use of an underrelaxation algorithm: let $\lambda_{n}$ be the value of some function we are solving for at the $n$th iteration. Then at iteration $n+1$ we underrelax $\lambda_{n+1}$ by setting

$$
\lambda_{n+1}=\lambda_{n}+q\left(\lambda_{n+1}-\lambda_{n}\right),
$$

where $0 \leq q \leq 1$. We found the best results by applying the above algorithm to both the metric potentials and the stress-energy tensor components. Typically, we used $q$ between 0.8 and 0.9 . Also, a significant number of iterations can be saved during a sequence of calculations in which the heating parameter $b$ is varied if we use the previous solution as the initial guess when we change the value of $b$.

For disks in the relativistic region, 600 radial zones, 20 angular zones, and 20 Legendre polynomials are adequate.

\section{INITIAL DATA}

As an initial guess for the metric potentials $\rho(r, \mu), \gamma(r, \mu)$, $\omega(r, \mu)$, and $\sigma(r, \mu)$, we used their Newtonian limits, given in

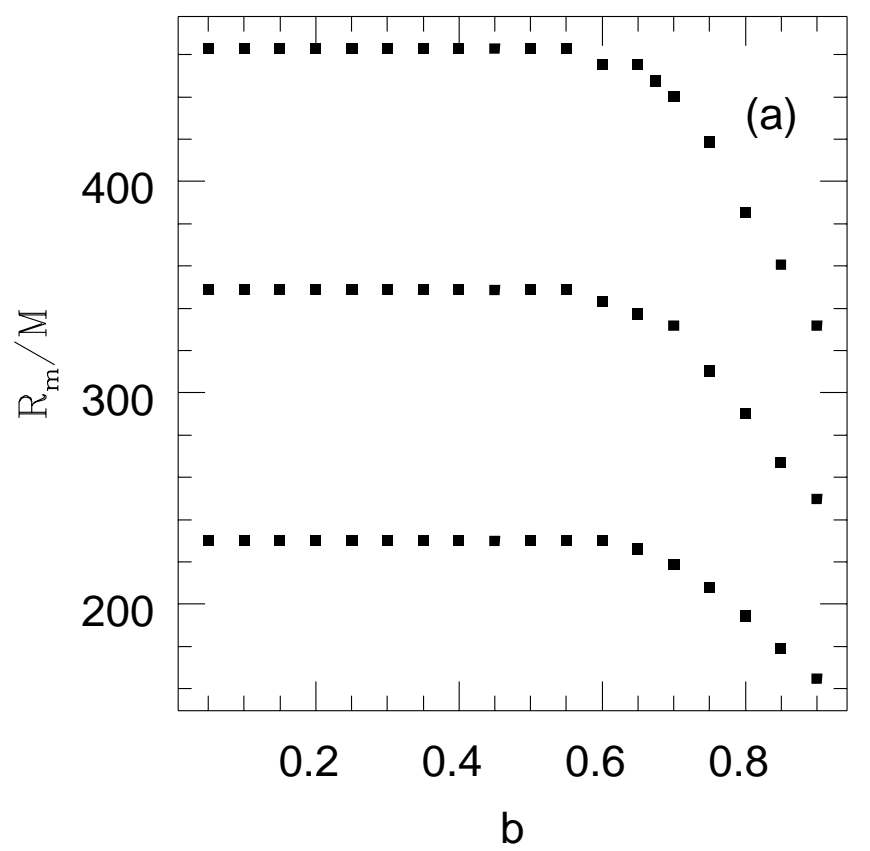

terms of the Newtonian potential $\Phi_{\mathrm{N}}$, by

$$
\begin{aligned}
\rho(r, \mu) & \rightarrow 2 \Phi_{\mathrm{N}}(r, \mu), \\
\gamma(r, \mu) & \rightarrow 0, \\
\sigma(r, \mu) & \rightarrow-\Phi_{\mathrm{N}}(r, \mu), \\
\omega(r, \mu) & \rightarrow 0 .
\end{aligned}
$$

Mihalas (1968) gives an expression for the Newtonian potential of an oblate homogeneous spheroid. By taking the limit as the eccentricity $e \rightarrow 1$, we obtain the Newtonian potential $\Phi_{\mathrm{N}}(r, \mu)$ for all points both on and off the disk. Outside the matter, we find

$$
\begin{aligned}
\Phi_{\mathrm{N}}(r, \mu)= & -\frac{3 M}{2 R_{m}}\left(B-\frac{q_{s}}{2\left(1-x^{2}\right)^{1 / 2}}\right. \\
& \left.\times\left\{\left(1-3 \mu^{2}\right)\left[B\left(1-x^{2}\right)^{1 / 2}-x\right]+\left(1-\mu^{2}\right) x^{3}\right\}\right),
\end{aligned}
$$

where $q_{s} \equiv r^{2} / R_{m}^{2}, x$, and $B=\arcsin x$, with $p$ defined by the quadratic equation

$$
q_{s}\left(1-\mu^{2}\right) p^{2}-\left(q_{s}+1\right) p+1=0 .
$$

Inside the matter, we have

$$
\Phi_{\mathrm{N}}(r, \mu)=-\frac{3 \pi M}{4 R_{m}}\left(1-\frac{q_{s}}{2}\right) .
$$

\section{NUMERICAL RESULTS}

Our region of investigation spans values of the heating parameter in the range $0.05 \leq b \leq 0.95$ for values of $R_{m} / M$ ranging from $R_{m} / M=455$ down to $R_{m} / M=6.26$. Figure $1 a$ shows the convergence of some representative runs in the Newtonian region. As we can see, as $b$ increases, $R_{m} / M$ of

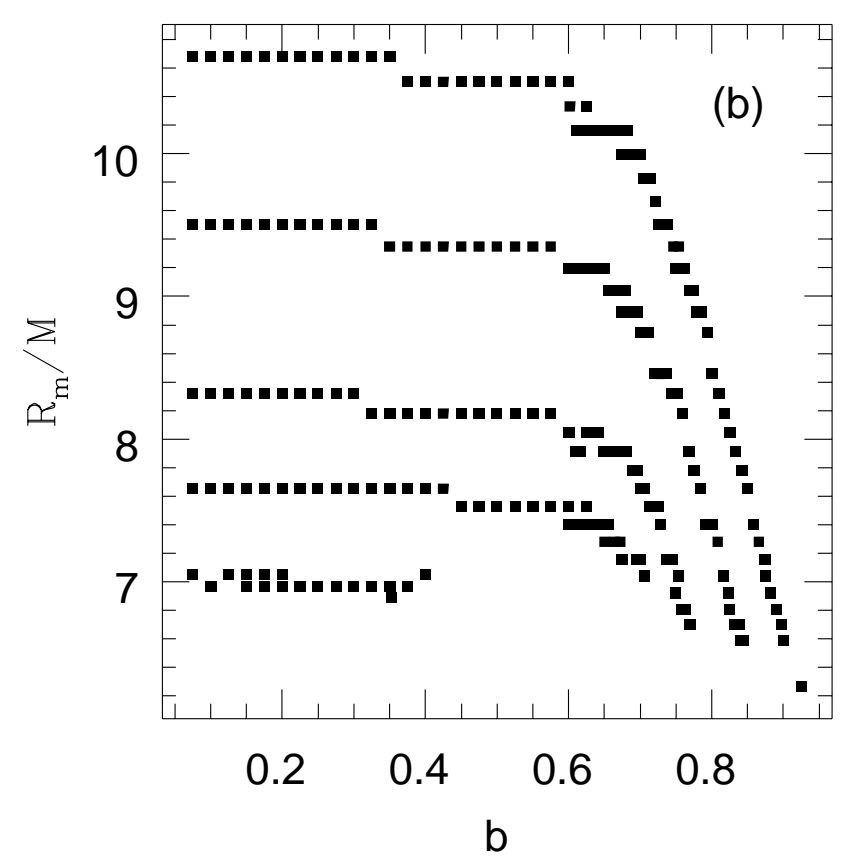

FIG. 1.-Convergent runs for some representative values of $R_{m} / M$. (a) Runs in the Newtonian region. (b) Runs in the more relativistic region. 
the solution decreases. This is also evident for the more relativistic cases shown in Figure $1 b$.

To better understand our solutions it is helpful to look at how the central redshift $z_{c}$ varies with the heating parameter $b$. For the Newtonian Kalnajs disk given in the Appendix, we can calculate the central redshift $z_{c}$ analytically from the Newtonian gravitational potential as follows:

$$
\begin{aligned}
z_{c} & \equiv\left[-g_{t t}(0,0)\right]^{-1 / 2}-1 \\
& =\left(e^{\gamma(0,0)+\rho(0,0)}\right)^{-1 / 2}-1 .
\end{aligned}
$$

Now use equation (68) to get

$$
z_{c}^{\text {Newtonian }}=-\phi_{\mathrm{N}}(0,0)=\frac{3 \pi M}{4 R} .
$$

This quantity is independent of the heating parameter $b$, and thus is a useful diagnostic. Although analytic equilibrium solutions exist in the Newtonian case even in the unstable region $(b \geq 0.816$; see the Appendix), it is not obvious that our iterative method will converge to them. Instabilities may be signaled by failure to converge, or by causing convergence to a solution that is far from the true Kalnajs solution. (Of course, a failure to converge does not necessarily imply the existence of an instability.) Thus by plotting the central redshift versus the heating parameter, we can hope to see where the solution we find differs from the true Newtonian solution. As can be seen in Figure 2a, for a Newtonian cluster with $R_{m} / M=455$, our code finds the Kalnajs equilibrium solutions for $b \leq 0.6$. Since the stability region is given by $0 \leq b<0.816$, the change in the central redshift does not serve as a sharp indicator of the transition to the unstable region.

Similar behavior can be observed in our relativistic clusters. Figure $2 b$ shows an example for $R_{m} / M=8.84$. Regions in which $z_{c}$ is not constant may represent true general relativistic equilibria, but most likely these clusters are near or inside the general relativistic unstable region. To fully pin down this region requires a dynamical stability analysis, which can probably only be done by numerical evolution.

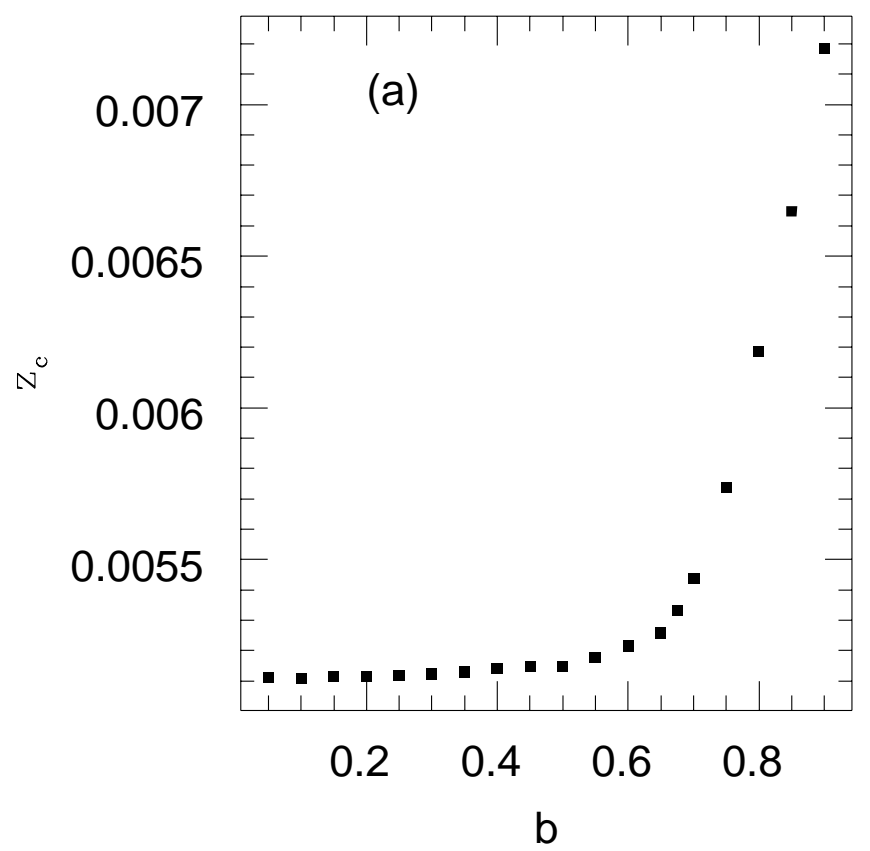

The most relativistic solution we have found has an $R_{m} / M$ of 6.91 . By comparing this disk to the Kerr geometry we can get an idea of how relativistic this model is. Using the proper circumference of the disk, we find that our most relativistic cluster corresponds to an $R / M$ for Kerr of 8.03. Table 1 gives some properties of these representative models. These data are from runs converged at the $1 \times 10^{-4}$ level.

In Figure 3 we show contour plots of the surface density for two examples of our equilibrium disks. Figure $3 a$ shows a representative Newtonian cluster $\left(R_{m} / M=455\right)$ with $b=0.6$. In Newtonian theory these disks have homogeneous volume density. Figure $3 b$ shows the same plot for the analytic version of the same cluster. In contrast, Figures $3 c$ and $3 d$ show a representative relativistic cluster $\left(R_{m} / M=6.97\right)$ with $b=0.1$ and its Newtonian counterpart, respectively. The relativistic disks are, in general, more centrally condensed than their Newtonian cousins.

In previous work (Shapiro \& Teukolsky 1985, 1992) on polytropic axisymmetric clusters, we found that a maximum in the binding energy along an equilibrium sequence marked the onset of a dynamical instability. Since for the Newtonian Kalnajs disks here the potential (eq. [A7]) and surface matter density (eq. [A4]) are not functions of the heating parameter, the binding energy is also not a function of $b$. Thus it cannot be used to analyze stability. However,

TABLE 1

Properties of Some RePresentative Solutions

\begin{tabular}{cccccl}
\hline \hline$R_{m} / M$ & $b$ & $z_{c}$ & $g_{t t}^{\operatorname{maxa}}$ & \multicolumn{1}{c}{$E_{b}{ }^{\mathrm{b}}$} & \multicolumn{1}{c}{$J^{\mathrm{c}}$} \\
\hline $452 \ldots \ldots$ & 0.6 & 0.0053 & -0.9896 & -0.0103 & 3.88 \\
$6.91 \ldots \ldots$ & 0.1 & 0.9560 & -0.2612 & $\ldots{ }^{\mathrm{d}}$ & 0.0690 \\
\hline
\end{tabular}

${ }^{\text {a }} g_{t t}^{\max }$ is the maximum value of $g_{t t}$.

${ }^{b} E_{b}$ is in units of $M_{0}$.

c $J$ is in units of $M^{2}$.

${ }^{\mathrm{d}}$ For the relativistic disk here our value of $E_{b}$ is unreliable because of numerical errors.

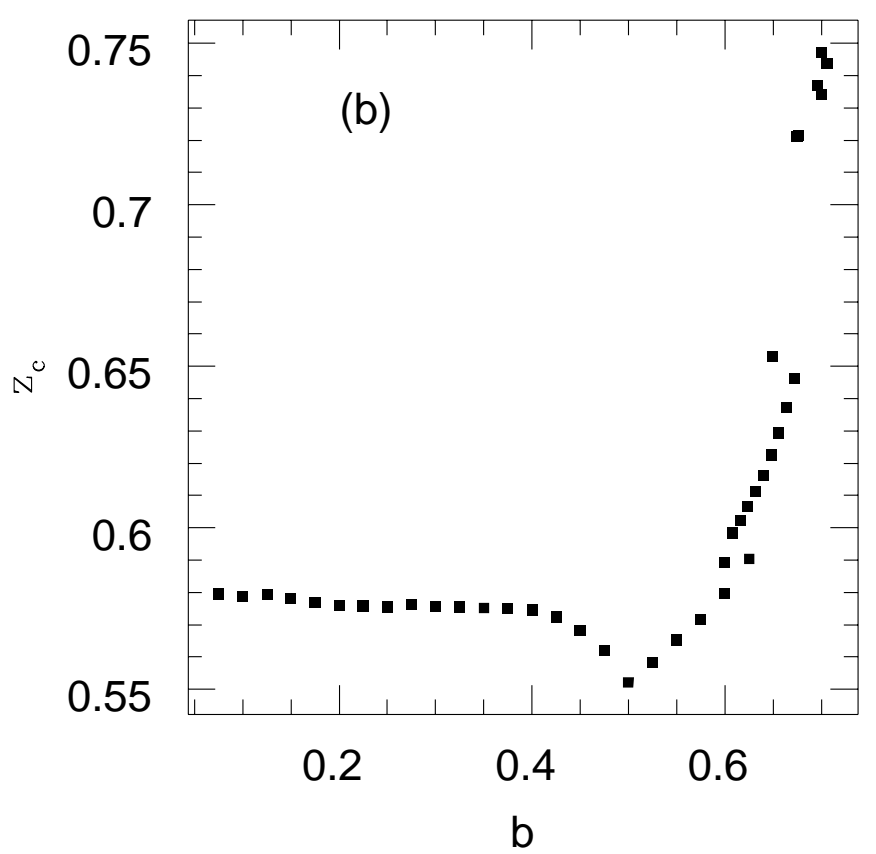

FIG. 2. Central redshift $\left(z_{c}\right)$ vs. heating parameter $b$ for a Newtonian cluster. $(a) R_{m} / M=455$ and a representative relativistic cluster. $(b) R_{m} / M=8.84$. 

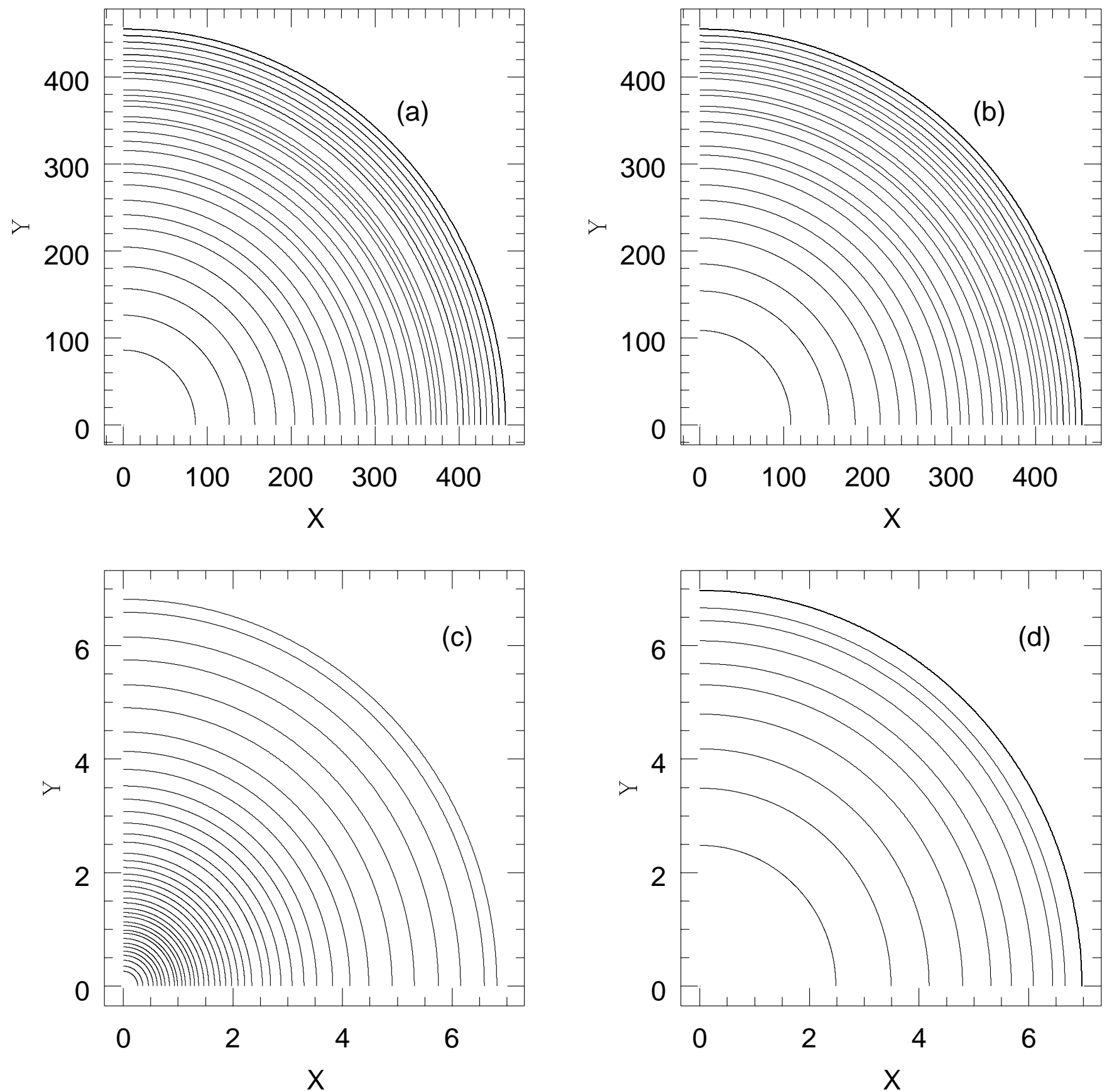

FIG. 3.-Surface density contour plots: (a) Newtonian cluster with $R_{m} / M=455$, and (b) its analytic analog. (c) A representative relativistic cluster with $R_{m} / M=6.97$. (d) The analytic Newtonian version of this cluster $\left(R_{m} / M=6.97\right)$.

in the general relativistic case we expect this degeneracy to be lifted. We do indeed find that the binding energy does depend on $b$. However, because of numerical errors we are not able to reliably find the turning point in the binding energy that would signal the onset of instability. Again, a firm limit on the stability region must wait for a full dynamical evolution code.
We thank Mark Scheel and Greg Cook for useful discussions. We also thank Greg Cook for generously sharing a computer code with us. This work has been supported in part by National Science Foundation grant PHY 94-08378 at Cornell University, and by National Science Foundation grant AST 96-18524 and NASA grant NAG 5-3420 at the University of Illinois at Urbana-Champaign. 


\section{APPENDIX}

\section{NEWTONIAN KALNAJS DISKS}

A Newtonian Kalnajs disk can be obtained by "flattening" a homogeneous oblate spheroid, i.e., by letting the eccentricity $e \rightarrow 1$ (Binney \& Tremaine 1987). This flattening yields a disk with a surface density given by

$$
\Sigma(R)=\Sigma_{c}\left(1-R^{2} / R_{m}^{2}\right),
$$

where $\Sigma_{c}$ is the central density, $R_{m}$ is the radius of the disk (matter radius), and $R$ is the radius in the disk plane defined by

$$
R \equiv r \sin \theta .
$$

When all of the particles are in circular orbits with uniform angular velocity $\Omega_{\text {circ }}$, then $\Omega_{\text {circ }}$ is related to the central density by the same relation as for Maclaurin spheroids.

Kalnajs (1972) developed a family of equilibrium disks, all of which have the above surface density. Each member of this family is characterized by the parameter $b$ defined in equation (35).

These disks have velocity dispersions that are governed by the tunable parameter $b$ :

$$
\overline{\left(v^{\hat{\phi}}\right)^{2}}-\left(\overline{v^{\hat{\phi}}}\right)^{2}=\overline{\left(v^{\hat{R}}\right)^{2}}=\frac{1}{3} V^{2}\left(1-b^{2}\right)\left(1-\frac{R^{2}}{R_{m}^{2}}\right) .
$$

From the above equation we can see that when $b \approx 1\left(\Omega R_{m} \approx V\right)$, these disks are "cold," i.e., have very little thermal motion. This corresponds physically to a system in which all particles move on nearly circular orbits. On the other hand, when $b \ll 1$ $\left(\Omega R_{m} \ll V\right)$, we have "hot" systems in which most of the support against self-gravity comes from the random motions given in equation (A3) (Binney \& Tremaine 1987).

A distribution function that generates this family is

$$
f_{\mathrm{N}}\left(E_{\mathrm{N}}, J_{z \mathrm{~N}}\right)= \begin{cases}K_{\mathrm{N}}\left\{2\left[\left(b V J_{z \mathrm{~N}} / R_{m}\right)-E_{\mathrm{N}}\right]-V^{2}\left(1+b^{2}\right)\right\}^{-1 / 2}, & {[\cdots]>0,} \\ 0, & {[\cdots] \leq 0 .}\end{cases}
$$

Here $K_{\mathrm{N}}$ is a constant that can be determined by fixing the total mass of the system, $E_{\mathrm{N}}$ is the Newtonian energy, and $J_{z \mathrm{~N}}$ is the Newtonian angular momentum about the symmetry $(z)$ axis. These quantities, with $m=1$, are given by

$$
\begin{gathered}
E_{\mathrm{N}}=\frac{\left(p^{\hat{R}}\right)^{2}}{2}+\frac{\left(p^{\hat{\phi}}\right)^{2}}{2}+\phi_{\mathrm{N}}(R) \\
J_{z \mathrm{~N}}=R p^{\hat{\phi}} .
\end{gathered}
$$

The Newtonian potential, $\phi_{\mathrm{N}}(R)$, in the disk plane is

$$
\phi_{\mathrm{N}}(R) \equiv \Phi_{\mathrm{N}}(r, 0)=-V^{2}\left(1-\frac{R^{2}}{2 R_{m}^{2}}\right)
$$

Using the linearized collisionless Boltzmann equation (linearized Vlasov equation), Kalnajs (1972) analyzed the stability of this family of disks. He found that restricting the heating parameter, $b$, to be less than 0.816 produced disk systems that are stable against all axisymmetric disturbances, i.e., disks with $0 \leq b<0.816$ are stable against ring formation.

\section{REFERENCES}

Abrahams, A. M., Shapiro, S. L., \& Teukolsky, S. A. 1994, Phys. Rev., 50, 7282

Bardeen, J. M. 1970, ApJ, 162, 71

Bardeen, J. M., \& Wagoner, R. V. 1969, ApJ, 158, L65

Binney, J., \& Tremaine, S. 1987, Galactic Dynamics (Princeton: Princeton Univ. Press), chap. 4

Cook, G. B., Shapiro, S. L., \& Teukolsky, S. A. 1992, ApJ, 398, 203

Fridman, A. M., \& Polyachenko, V. L. 1984, Physics of Gravitating

Systems (New York: Springer)

Kalnajs, A. J. 1972, ApJ, 175, 63

Kley, W. 1997, MNRAS, 287, 26

Komatsu, H., Eriguchi, Y., \& Hachisu, I. 1989, MNRAS, 237, 355

Lightman, A. P., Press, W. H., Price, R. H., \& Teukolsky, S. A. 1975,

Problem Book in Relativity and Gravitation (Princeton: Princeton

Univ. Press)
Mihalas, D. 1968, Galactic Astronomy (San Francisco: Freeman)

Morgan, T., \& Morgan, L. 1969, Phys. Rev., 183, 1097

Neugebauer, G., \& Meinel, R. 1993, ApJ, 414, L97

. 1994, Phys. Rev. Lett., 73, 2166 1995, Phys. Rev. Lett., 75, 3046

Press, W. H., Teukolsky, S. A., Vetterling, W. T., \& Flannery, B. P. 1992, Numerical Recipes in Fortran: The Art of Scientific Computing (2d ed.; Cambridge: Cambridge Univ. Press)

Salpeter, E. E., \& Wagoner, R. V. 1971, ApJ, 164, 557

Shapiro, S. L., \& Teukolsky, S. A. 1985, ApJ, 298, 58

. 1992, Philos. Trans. R. Soc. London, A, 340, 365 1993, ApJ, 419, 636 